\title{
Idiopathic Necrotizing Granulomas of the Thyroid: Report of a Rare Case
}

\author{
Anupam $\mathrm{K}^{1,2}$, Jason $\mathrm{DE}^{2}$, Diva $\mathrm{RS}^{3}$ and Vahab $\mathrm{F}^{1,2 *}$ \\ ${ }^{1}$ Division of Endocrinology, Diabetes, Metabolism, and Nutrition, Mayo Clinic, USA \\ ${ }^{2}$ Department of Medicine, Mayo Clinic, Rochester, USA \\ ${ }^{3}$ Department of Laboratory Medicine and Pathology, Mayo Clinic, Rochester, USA
}

*Corresponding author: Vahab Fatourechi, MD, Professor of Medicine, Division of Endocrinology, Diabetes, Metabolism, and Nutrition, Department of Medicine, Mayo Clinic, 200 First Street SW, Rochester, New York, USA, MN 55905

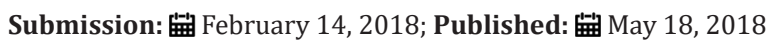

\begin{abstract}
Granulomatous disease of the thyroid commonly presents as sub acute (De Quervain) thyroiditis [1]. Necrotizing granulomas in thyroid have been reported with infection (tuberculous [2] and fungal [3]), granulomatosis with polyangiitis [4], plasma cell granuloma[5], post-surgical/palpation thyroiditis [6] and rarely in sarcoidosis (usually causes non-necrotizing granulomas). We present a case of necrotizing granulomas within thyroid of unclear etiology associated with sudden profound hypothyroidism.
\end{abstract}

Keywords: Necrotizing granulomas; Hypothyroidism; Tuberculosis; Subacute thyroiditis

\section{Case presentation}

A 75 year old male presented to his physician with 6 months of fatigue, poor concentration, cold intolerance, generalized weakness and gait imbalance. He denied neck pain, preceding viral illness or hyperthyroid symptoms. Palpation revealed anon-tender stony hard thyroid. His TSH was $153 \mathrm{mIU} / \mathrm{L}$ (reference $0.3-4.2$ ) as compared to a normal level 6 months prior; and free T4 was $0.3 \mathrm{ng} / \mathrm{dL}$ (reference $0.9-1.7)$. Thyroid peroxidase and thyro globulin antibodies were not elevated. He underwent partial left lobe resection at another institution which on histology showed necrotizing granulomas with multinucleated giant cells on a background of fibrosis and chronic lymphocytic inflammation (Figure 1A). QuantiFERON Gold and PPD skin tests for Tuberculosis were negative. Levothyroxine 100 micrograms daily was initiated for primary hypothyroidism, however the etiology of granulomas remained a mystery.

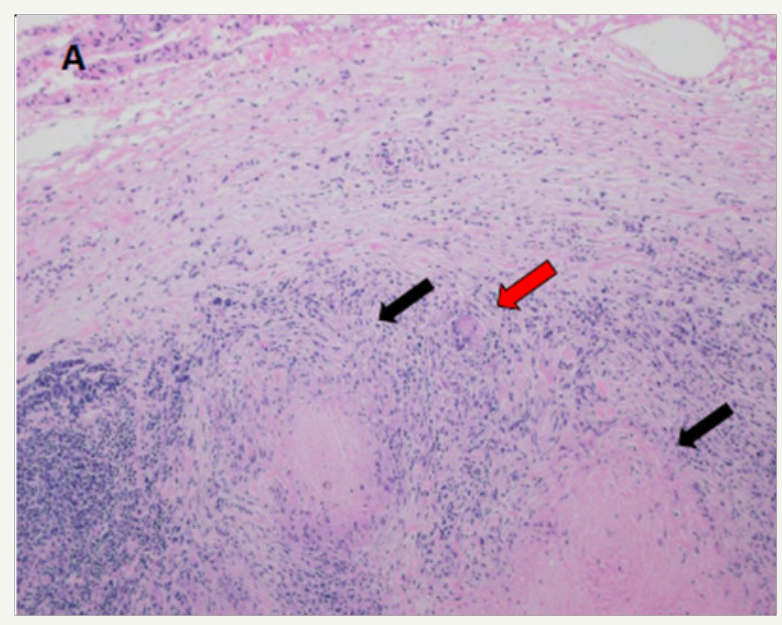

Figure 1A: Partial left thyroid lobe resection histology with hematoxylin and eosin stain original magnification $\mathrm{x} 40$ showing complete effacement of thyroid parenchyma by the presence of background fibrosis and chronic lymphocytic inflammation. Necrotizing granulomas (black arrows) and a few multinucleated giant cells (red arrow) are observed. 
Over the next 6 months, his symptoms of fatigue, poor concentration and cold intolerance improved, however weakness and gait imbalance persisted. This prompted him to present to Mayo Clinic for evaluation by Endocrinology and Neurology. Examination demonstrated a small, firm thyroid without nodularity or tenderness, and marked proximal muscle weakness and atrophy. Laboratory evaluation showed a normal TSH of $1.9 \mathrm{mIU} / \mathrm{L}$ and free of T4 of $1.8 \mathrm{ng} / \mathrm{dL}$ while taking levothyroxine. Ultra sound show small, diffusely hypoechoic (Figure 1B) and hypovascular (Figure 1C) thyroid without nodules. Workup for pertinent autoimmune conditions including anti-nuclear antibody, rheumatoid factor,
anti-Smith antigen, anti-ribonucleo protein, anti-Scl 70, anti-Jo 1, anti-myeloperoxidase, anti-proteinase 3 and immunoglobulin G4 (IgG4) resulted negative.ESR and CRP were normal.Core biopsyof right thyroid lobe demonstrated histological features similar to the previous specimen (Figure 1D). Tissue stains and cultures were negative for bacteria, mycobacteria, fungi or elevated IgG4 staining cells. Levothyroxine was continued and statin dose decreased for possible contribution to myopathy. Eight monthslater, muscle strength improved, and he remained clinically and biochemically euthyroid on levothyroxine replacement.

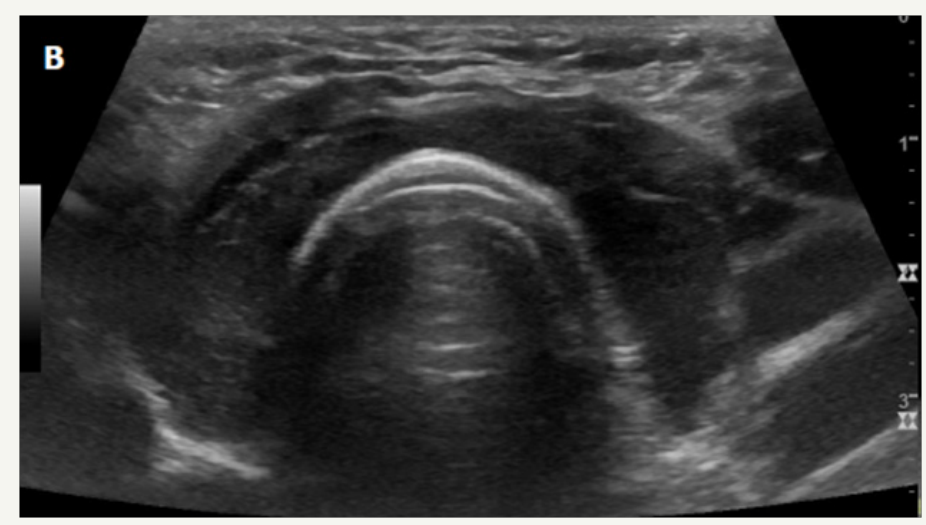

Figure 1B: Ultrasound showing a diffusely hypoechoic thyroid gland on transverse view.

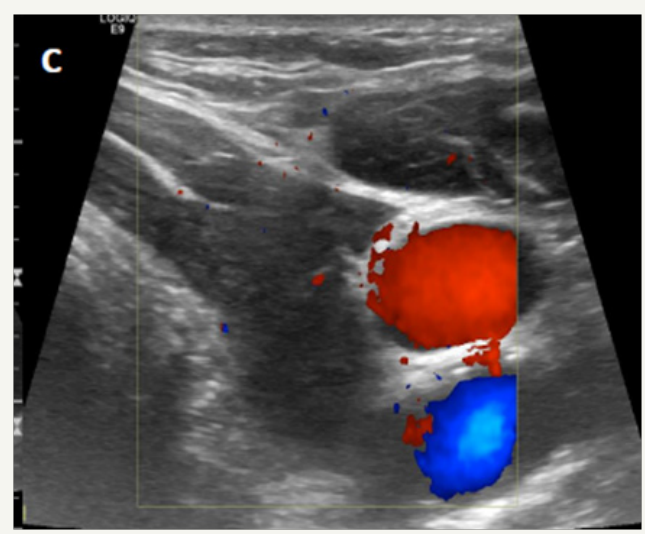

Figure 1C: Ultrasound with Doppler showing hypovascular left thyroid lobe on transverse view.

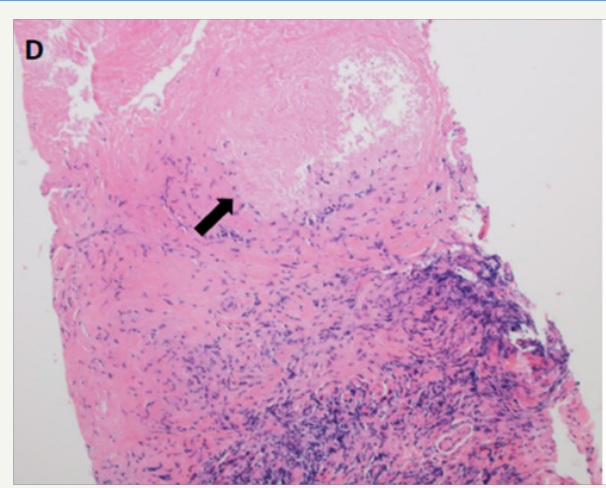

Figure 1D: Right lobe core biopsy histology with hematoxylin and eosin stain original magnification $\mathrm{x} 100$ showing necrotic granuloma (black arrow) surrounded by vague rim of palisading histiocytes. The remainder of the tissue shows fibrosis and chronic inflammation. 


\section{Discussion}

This case was differentiated from subacute thyroiditis by the absence of pain, preceding thyrotoxicosis phase or upper respiratory illness. It was differentiated from other causes of necrotizing granulomas by negative evaluation for pertinent autoimmune, infiltrative and infectious causes. The myopathy was felt to be multifactorial due to statin use and hypothyroidism. An extensive search of Mayo Clinic records from 1997-2017 and available literature did not yield any cases of similar pathology without a clear etiology involving the thyroid; however, this has been reported in other organs like lungs [7].

\section{Conclusion}

To conclude, this case of necrotizing granulomas within thyroid gland causing sudden debilitating hypothyroidism would be considered idiopathic in etiology, the first such reported case to our knowledge. We recommend core biopsyto aid in the diagnosis of certain infiltrative, autoimmune and infectious diseases of the thyroid.

\section{Objective}

The objective of reporting this case is to highlight the importance of core biopsy to aid in the diagnosis of certain thyroid disorders; however, the exact etiology may remain uncertain in some cases.

\section{References}

1. Meachim G, Young MH (1963) De Quervain's subacute granulomatous thyroiditis: histological identification and incidence. Journal of Clinical Pathology 16(3): 189-199.

2. Ozekinci S, Mizrak B, Saruhan G, Senturk S (2009) Histopathologic diagnosis of thyroid tuberculosis. Thyroid 19(9): 983-986.

3. Moinuddin S, Barazi H, Moinuddin M (2008) Acute blastomycosis thyroiditis. Thyroid 18(6):659-661.

4. Schmitz KJ, Baumgaertel MW, Schmidt C, Sheu SY, Betzler M (2008) Wegener's granulomatosis in the thyroid mimicking a tumour. Virchows Archiv 452(5): 571-574.

5. Barber WA, Fernando M, Chadwick DR (2010) Plasma Cell Granuloma of the Thyroid: A Conservative Approach to a Rare Condition and Review of the Literature. Journal of Thyroid Research 840469.

6. Carney JA, Moore SB, Northcutt RC, Woolner LB, Stillwell GK (1975) Palpation Thyroiditis (Multifocal Granulomatous Folliculitis). American Journal of Clinical Pathology 64(5): 639-647.

7. Aubry MC (2012) Necrotizing granulomatous inflammation: what does it mean if your special stains are negative [quest]. Mod Pathol 25(S1): S31-S8.
Creative Commons Attribution 4.0 International License

For possible submissions Click Here
Submit Article

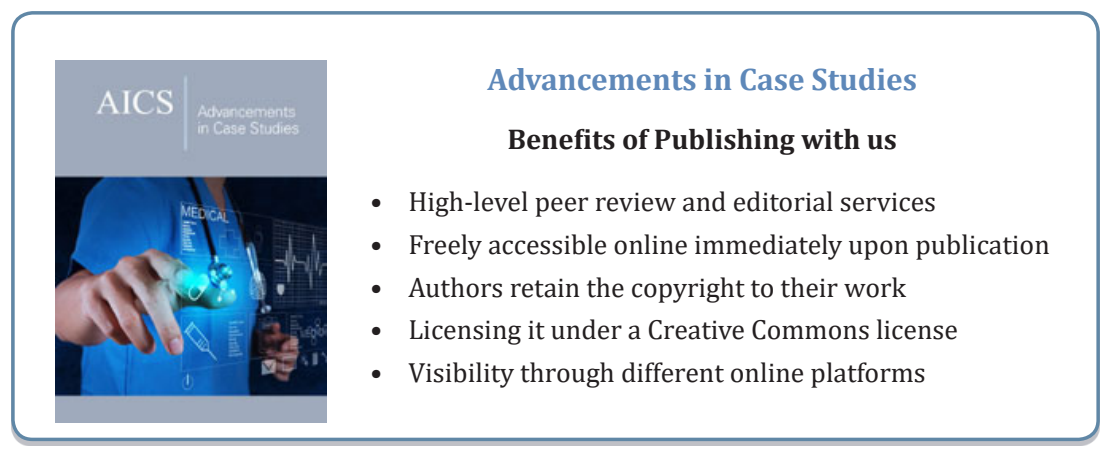

